\title{
Remediation of context-processing deficits in schizophrenia: preliminary data with ambiguous
} \section{sentences}

\author{
Chrystel Besche-Richard ${ }^{1,2}$ \\ Sarah Terrien' \\ Marion Lesgourgues ${ }^{3,4}$ \\ Célia Béchiri-Payet ${ }^{5}$ \\ Fabien Gierski ${ }^{1,3}$ \\ Frédéric Limosin ${ }^{6-8}$ \\ 'Laboratory Cognition, Santé, \\ Socialisation, University of Reims \\ Champagne-Ardenne, France; ${ }^{2}$ Institut \\ Universitaire de France, Paris, France; \\ ${ }^{3}$ Centre Hospitalier Universitaire, \\ Pôle de Psychiatrie des Adultes, \\ Reims, France; ${ }^{4}$ Service Universitaire \\ de Médecine Préventive et de \\ Promotion de la Santé, University \\ of Reims Champagne-Ardenne, \\ Reims, France; ${ }^{5}$ Etablissement Public \\ de Santé mentale départemental \\ de l'Aisne, Prémontré, France; \\ ${ }^{6}$ Department of Adult and Geriatric \\ Psychiatry, Hôpitaux Universitaires \\ Paris, Ouest (Assistance publique- \\ Hôpitaux de Paris), Hôpital Corentin- \\ Celton, Issy-les-Moulineaux, France; \\ ${ }^{7}$ Faculty of Medicine, University \\ Paris Descartes, Sorbonne Paris \\ Cité, Paris, France; ${ }^{8}$ Psychiatry \\ and Neurosciences Center, French \\ National Institute of Health and \\ Medical Research, Institut National de \\ la Santé et de la Recherche Médicale \\ U894, Paris, France
}

Correspondence: Chrystel BescheRichard

Laboratory Cognition, Santé,

Socialisation, C2S, EA629I, Université

de Reims Champagne-Ardenne,

57 Rue Pierre Taittinger, 51096

Reims, Cedex, France

$\mathrm{Tel}+33326913776$

Fax +33 3 269l 37I9

Email chrystel.besche@univ-reims.fr
This article was published in the following Dove Press journal:

Neuropsychiatric Disease and Treatment

3 December 2014

Number of times this article has been viewed

Background: Processing of contextual information is essential for the establishment of good interpersonal relations and communicational interactions. Nevertheless, it is known that schizophrenic patients present impairments in the processing of contextual information. The aim of this study is to explore the influence of the remediation of context processing in schizophrenic patients.

Methods: Thirty-one schizophrenic patients and 28 matched healthy participants were included in this study. All participants were assessed on verbal knowledge (Mill-Hill test) and depression intensity (Beck Depression Scale 21 items). Schizophrenic patients were also assessed on thought, language, and communication disorders (Thought, Language and Communication scale). All participants completed a disambiguation task with two different levels of contextualization (high or low context) and a context-processing remediation task containing social scenarios that included ambiguous words and were presented with two different types of instruction: with or without context explanation.

Results: For the disambiguation task, results showed no effect of group, but a main effect of context, with better performances in the high-context than the low-context condition. For the context-processing remediation task, results showed a main effect of group: The performance of schizophrenic patients who had received explanations differed from that both of healthy participants and of schizophrenic patients who had not received explanations.

Conclusion: This study revealed that for all participants, the structuring of context had a positive effect on the contextual integration of ambiguous words. Concerning the remediation task, explanations about the strategies that could be used to take context into account improved the schizophrenic patients' performances. This allows us to consider new methods of remediation that could improve social interaction in schizophrenia.

Keywords: schizophrenia, context information, remediation, semantic, subordinate meaning

\section{Introduction}

Contextual information is considered to be crucial for interaction with our overall environment in different domains such as perception and literal and figurative language (more specifically, semantics), as well as social cognition and theory of mind. ${ }^{1}$ McClure et $\mathrm{al}^{2}$ defined context information as "information actively maintained in such a form that it can be used to mediate later task-appropriate behavior" (p. 343). Without the processing of contextual information, interpersonal relations and communicational interactions frequently fail and, more generally, behavior is not adapted to the situation and could lead to social impairments. Most of the cognitive disorders identified in schizophrenia could result from an impairment in the processing of contextual information. ${ }^{3}$ Indeed, this deficit in contextual processing has been found in tasks involving perception and attention (Continuous Performance Test-Form AX), ${ }^{4,5}$ memory, ${ }^{3,6}$ and language (as is explained in more detail in this introduction). Moreover, 
a failure to process contextual information or an inefficiency in constructing and maintaining internal representations of context have frequently been proposed as hypotheses capable of explaining the social and communication disorders observed in schizophrenic patients. ${ }^{7,8}$ Because of the difficulties they experience in taking account of contextual information, schizophrenic patients present some discourse ruptures during communication with others that, at a clinical level, are considered to reflect thought and language disorders. ${ }^{9}$ They also exhibit difficulties in processing a special context; namely, the mental states of others. ${ }^{10}$

At an experimental level in the field of language research, these contextual information impairments have usually been studied by means of semantic priming tasks, which are a good index of contextual processing. ${ }^{11}$

Many studies have used the semantic priming paradigm in schizophrenic patients to support the hypothesis of a dysfunctional processing of contextual information. Although the data are not always consistent, they suggest that schizophrenic patients, and particularly schizophrenic patients with high levels of thought disorders, find it difficult to take semantic context into account. This is possibly a result of either the persistence of a hyperactivation, in semantic memory, of the prime's representation and its associates via semantic nodes, followed by a difficulty in inhibiting the irrelevant information (such as activated associated words that are not presented as the target; eg, Pomarol-Clotet et $\mathrm{al}^{12}$ ), or a difficulty in matching the prime (context) and the target to facilitate the decision when the prime and target are related (eg, Besche-Richard and Passerieux ${ }^{13}$ ).

More specifically, linguistic situations necessitate the processing of contextual information in the presence of ambiguous or polysemic words, which are specific in having at least two meanings; namely, a dominant and a nondominant (subordinate) sense. Access to the nondominant meaning of an ambiguous word is possible thanks to the comprehension and integration of its context of occurrence. For example, in the phrase "When the farmer bought a herd of cattle, he needed a new pen," "14 the dominant meaning of "pen" is not activated or is inhibited in favor of the subordinate meaning, as required by the context of occurrence of this ambiguous word.

The first studies of the processing of contextual information in schizophrenic patients used ambiguous words and revealed how difficult it is for patients to process the nondominant meaning of a word. This is because they ignore the context of occurrence: Independent of the context, schizophrenic patients interpret ambiguous words with reference to their dominant meanings. ${ }^{15}$ More recently, a set of studies has confirmed the existence of a dominant bias in schizophrenic patients..$^{3,14,16-18}$ Interestingly, Titone et $\mathrm{al}^{14}$ showed that more strongly biased contextual information is necessary if schizophrenic patients are to be able to inhibit material that is contextually irrelevant. This result drew attention to the possibility that more structured situations might favor improved comprehension of the subordinate meaning of ambiguous words and raises the question of whether there are any experimental conditions that might help reduce the difficulties experienced by schizophrenic patients when processing ambiguous words. For example, Besche-Richard and Passerieux ${ }^{13}$ have shown how the instructions (explicit versus implicit) and the proportion of related words (high versus low) during a lexical decision task tend to normalize semantic priming performance in schizophrenic patients. This could be a way of achieving the cognitive remediation of context-processing deficits in schizophrenia. In the field of cognitive remediation, several methods have been proposed to compensate for the cognitive impairment of executive functions, recognition of emotions, autobiographical memory, social cognition, and in particular, theory of mind. ${ }^{3,19-21}$ However, to the best of our knowledge, no study has investigated the remediation of contextual processing impairments in schizophrenic patients.

The aim of this study was to evaluate whether, for schizophrenic patients, a strong context (disambiguation task) or context-oriented instructions (context-processing remediation task) lead to better attribution/comprehension of the subordinate meaning of an ambiguous word presented in a sentence context (disambiguation task) or social scenario (context-processing remediation task). We formulated two main hypotheses. First, when the sentence context is neutral (low context), schizophrenic and healthy participants should prefer the dominant meaning of each ambiguous word. In contrast, when the sentence context is highly structured (high context) in favor of the subordinate meaning, schizophrenic and healthy participants should choose the nondominant meaning of each ambiguous word that is appropriate to the context. Second, when the explicit instructions outline the nature of the ambiguous scenario and guide the strategies employed to comprehend these ambiguous social situations, schizophrenic patients should perform better and exhibit a better comprehension of social situations containing ambiguous words than when no explicit instructions are given.

\section{Materials and methods Participants}

A total of 59 participants aged from 18 to 60 years were included in this study, including 31 neuroleptic-treated patients who met the Diagnostic and Statistical Manual of 
Mental Disorders, Fourth Edition, Text Revision (DSMIV-TR; APA 2000) $)^{22}$ criteria for schizophrenia and who were recruited from the Department of Psychiatry of Reims University Hospital in France. None of the patients suffered from any past or present neurological or somatic disease or alcohol or drug abuse or dependence, and none had received electroconvulsive therapy within the 6 months preceding the study. Twenty-eight healthy participants, matched with the patients on age, sex, and education level, and with no current or previous psychiatric disorders, including alcohol or drug abuse/dependence, were recruited from the general population.

All the healthy participants and schizophrenic patients were native French speakers and gave their written informed consent after the research had been explained to them. The study was designed in accordance with the Declaration of Helsinki.

All the participants were evaluated on verbal knowledge with the Mill-Hill part B ${ }^{23}$ to control for level of vocabulary, and on depression intensity with the Beck Depression Scale 21 items (BDI). ${ }^{24}$ The BDI is the self-report questionnaire most frequently used to evaluate the severity of depression. Each of the 21 items is rated on a 4-point Likert scale. The thought, language, and communication scale (TLC) ${ }^{25,26}$ was used to evaluate formal thought disorders in the schizophrenic patients. The TLC has traditionally been used to evaluate the severity of thought disorders in schizophrenia. It consists of 18 items (half of which are rated on a 3-point Likert scale and the other half on a 4-point Likert scale) and takes the form of a conversation between patient and interviewer. The total score yields information on thought and communication disorders. All the participants completed two linguistic tasks: a disambiguation task and a context-processing remediation task.

\section{Measures and procedure}

The first task was a disambiguation task with 20 aurally presented sentences, all of which contained a homograph used with its subordinate meaning. Twenty-six homographs were chosen from a dictionary of homographs ${ }^{27}$ and selected after a pretest involving 103 psychology students. Each homograph was presented accompanied by two pictures (one corresponding to the dominant meaning and the other to the nondominant meaning), and the participants had to choose the picture corresponding to the most frequent meaning of the word. We retained 20 homographs whose dominant meanings were selected in at least $70 \%$ of cases and whose secondary meanings were selected in no more than $30 \%$ of cases. Ten homographs were presented in a highly structured context ("Sophie vérifie son maquillage dans la glace de la salle de bain"/“Sophie checks her make-up in the bathroom mirror/ice cream"), and ten other homographs were presented in a weakly structured context ("Cette dame voudrait qu'on lui décrive le patron"/"This woman wants someone to describe the pattern/boss"). The sentences were presented using E-Prime ${ }^{\circledR}$ software and were spoken aloud by a female voice. After the sentences had been listened to, two pictures were presented $(420 \times 360$ pixels): one associated with the dominant meaning of the ambiguous word and the other with the secondary meaning. The participant had to choose which of the two pictures corresponded to the meaning of the sentence. The sentences were counterbalanced across two experimental lists so that both of them were presented in the two contexts. The dependent variable was the number of correct responses when the correct choice was the sentence corresponding to the subordinate meaning of the word in high and low context (the maximum score was 10 ).

The second task was the context-processing remediation task and contained ten interactive social scenarios presented in 20-second video sequences involving two persons during a social interaction. Each story ended with a sentence containing an ambiguous word (different from the words used in the disambiguation task). Two types of instructions guided the interpretation of the scenarios: either instructions encouraging the participants to pay attention to contextual elements (condition with explanation) or instructions that did not indicate any particular strategy (condition without explanation).

After a distractor task (Mill-Hill), an ambiguous question that contained the polysemic word and required the participant to take account of the context was asked. The schizophrenic patients were randomly (one patient of every two in each condition) assigned to one of the two groups: one benefiting from the condition with explanation and the other receiving no explanation. All the healthy participants were assigned to the condition without explanation. The dependent variable was the number of response errors in which the dominant meaning of each ambiguous word was chosen.

The order of presentation of the experiments was fixed, with the disambiguation task being completed first, followed by the context-processing remediation task.

\section{Statistical analysis}

Sociocultural and clinical data are presented in Table 1 . We first used two-tailed $t$-tests and $\chi^{2}$ when appropriate, to compare the demographic characteristics of the schizophrenic and healthy participants. We then analyzed the performances of the schizophrenic and healthy participants on the disambiguation task, using a repeated-measures analysis of variance (ANOVA) on the mean number of correct choices (ie, selection of the subordinate meaning of ambiguous words), with context (low context versus high context) as a within-group factor and group 
Table I Sociocultural and clinical characteristics (mean \pm standard deviation)

\begin{tabular}{|c|c|c|c|c|}
\hline Characteristics & Healthy participants, $n=28$ & Schizophrenic participants, $n=3$ I & $\chi^{2} / t$ & $P$ \\
\hline Sex, male/female & $23 / 5$ & $27 / 4$ & 0.28 & 0.59 \\
\hline Age, years & $37.2 \pm 9.9$ (range, $18-60)$ & $36.8 \pm 9$ (range, 18-60) & -0.17 & 0.85 \\
\hline Years of education & $8.3 \pm 6.7$ (range, $10-12$ ) & $10.8 \pm 6.5$ (range, $10-12$ ) & 1.6 & 0.12 \\
\hline Mill-Hill score & $33.7 \pm 5.3$ & $3 I .1 \pm 5.7$ & -1.8 & 0.08 \\
\hline Beck Depression Scale 2I, total score & $4.5 \pm 6.3$ & $8.4 \pm 6.9$ & 2.2 & $<0.03$ \\
\hline Thought, language, and communication total score & - & $6 \pm 4.9$ & - & - \\
\hline
\end{tabular}

(schizophrenic versus healthy participants) as a between-group factor. For the context-processing remediation task, we used a one-way ANOVA with the three groups (schizophrenic patients with context-oriented instructions, schizophrenic patients without specific instructions, and healthy participants) conducted on the number of errors (ie, when the dominant meaning of the words was chosen). In both tasks, analyses of covariance were carried out to control for the potential effect of confounding variables such as the Mill-Hill part B score, on the one hand, because vocabulary level can influence the comprehension of ambiguous words, and the BDI score, on the other hand, because schizophrenics and control patients are not matched on this dimension. Finally, we used correlations ( $r$ Bravais-Pearson) to test possible relations between the data from the two tasks and the severity of thought disorders measured by the TLC scale. Differences were considered to be statistically significant at a level of 0.05 . The data were analyzed using SPPS version 20 .

\section{Results}

\section{Demographics}

There were no significant differences on sociocultural variables (sex, age, level of education) between the schizophrenic and healthy participants. However, we found a marginal effect on verbal knowledge, with schizophrenic patients having lower scores (Table 1).

\section{Disambiguation task}

The repeated-measures ANOVA indicated a main effect of context $[F(1,57)=919.1 ; P<0.001]$, suggesting all participants exhibited better performances when the context was highly structured than when it was weakly structured. There were no effects of group $(F<1)$ and no significant interaction between context and group $(F<1)$. The means and standard deviations of the two groups are displayed in Table 2.

The same analysis conducted first with the Mill-Hill part B score and then with the BDI score as covariate (analyses of covariance) revealed comparable results. With the MillHill part B score, we found a significant effect of context $[F(1,56)=11.3 ; P<0.001]$, no effect of group $(F<1)$, and no significant interaction $(F<1)$; with the BDI score, we found a significant effect of context $[F(1,56)=512.01 ; P<0.0001]$, no effect of group $(F<1)$, and no significant interaction $(F<1)$.

\section{Context-processing remediation task}

Fifteen patients were assigned to the group with explanation, and 16 patients and all the healthy participants were assigned to the group without explanation. The two subgroups of patients did not differ in sex, age, level of education, BDI total score, or TLC total score, but only on Mill-Hill score $(\mathrm{t}[29]=-2.32 ; P<0.03)$, with the schizophrenic patients included in the group with explanation achieving higher scores (33.4 versus 28.9).

The one-way ANOVA revealed a main effect of group $(F[2,56]=46.7 ; P<0.001)$. Least squares difference post hoc tests showed that the error rates of the schizophrenic patients who received context-oriented instructions differed significantly both from those of the healthy participants $(P<0.01)$ and from those of the schizophrenic patients who did not receive explanations $(P<0.001$; see Table 3$)$. The healthy participants differed significantly from the schizophrenic patients who did not receive explanations $(P<0.001)$.

The same analysis conducted first with the Mill-Hill part B score and then with the BDI score as covariate (analyses of covariance) revealed comparable results: With the Mill-Hill part B score, we found a significant effect of group $[F(2,55)=37.1 ; P<0.0001]$, as we also did with the BDI score $[F(2,55)=43.4 ; P<0.0001]$.

\section{Correlation analysis}

There were no significant correlations between TLC scores and the results on the disambiguation task or the contextprocessing remediation task.

Table 2 Results of schizophrenic and healthy participants in the disambiguation task

\begin{tabular}{lll}
\hline Context & Healthy participants & Schizophrenic participants \\
\hline High & $9.96 \pm 0.2$ & $9.90 \pm 0.3$ \\
Low & $3.25 \pm 1.8$ & $3.32 \pm 1.4$ \\
\hline
\end{tabular}

Notes: Means ( \pm standard deviations) of the number of correct responses favoring the subordinate meaning of ambiguous words in the high-context and low-context conditions. 
Table 3 Mean of error rates ( \pm standard deviations) in the remediation task in the three groups of participants

\begin{tabular}{llll}
\hline & $\begin{array}{l}\text { Healthy } \\
\text { participants }\end{array}$ & $\begin{array}{l}\text { Schizophrenics } \\
\text { without explanation }\end{array}$ & $\begin{array}{l}\text { Schizophrenics } \\
\text { with explanation }\end{array}$ \\
\hline Error rates & $0.7 \mathrm{I} \pm \mathrm{I} .36$ & $5.56 \pm \mathrm{I} .75$ & $2.07 \pm \mathrm{I} .87$ \\
\hline
\end{tabular}

\section{Discussion}

The aim of this study was to evaluate, with different experimental manipulations (sentence structure level in the disambiguation task, instruction structure level in the context-processing remediation task), whether the structuring of context can improve the processing of ambiguous words inserted in the semantic context of sentences (disambiguation task) and in interactive and social semantic contexts (context-processing remediation task). The results of the disambiguation task revealed that the structuring of context had an effect on the probability of choosing a picture associated with the dominant and nondominant meanings of ambiguous words in each sentence. In effect, in the high-context condition, the participants chose the picture associated with the subordinate meaning of ambiguous words significantly more frequently than in the low-context condition. This effect was observed in all the participants, and there was no difference between the healthy and schizophrenic participants. We observed no group effect, with there being no significant difference between the performances of the schizophrenic patients in the high- and low-context conditions.

The results of the remediation task, which made use of interactive social scenarios containing ambiguous words, showed that the provision of explanations about the strategies used to process the context improved the schizophrenic patients' performances compared with those of the patients who received no explanations. These results are consistent with those of Besche-Richard and Passerieux ${ }^{13}$ and BescheRichard et al ${ }^{28}$ who showed that the reinforcement of semantic context (with a high proportion of semantically related words and explicit instructions to process the prime as a context) during lexical decision tasks with semantic priming increased the ability of schizophrenic patients to take account of the semantic context at the time of lexical decision. Our results are also compatible with those of Kayser et $\mathrm{al}^{29}$ who used short video sequences taken from French cinema movies showing interactions between two or more persons. Over a period of several sessions, a therapist taught the patients to analyze situations on the basis of their contextual environments and in the light of the intentions and mental states of the characters. This training significantly improved intention attribution performance in the schizophrenic patients who received the training compared with those who did not.
Overall, these results emphasize the possibility of remediating several of the cognitive disorders of schizophrenic patients by structuring the semantic, social, or environmental context.

Nevertheless, our results must be interpreted in the light of the fact that the schizophrenic group that received explanations achieved better verbal performances on the Mill-Hill test than the schizophrenic group that did not receive explanations, whereas the analysis of covariance using the Mill-Hill part B as covariate provided identical results, indicating that the schizophrenic patients included in the group with explanations about the strategies used to process the context achieved better results.

The first main limitation of this research concerns the absence of neuropsychological evaluation, and particularly of cognitive processes such as flexibility, inhibition, and the maintenance of context in working memory. These processes are potentially implicated in the processing of contextual information, including in the two tasks used in this research. In the disambiguation task and context-processing remediation task, participants must inhibit the dominant meaning of the ambiguous words and maintain the context in memory to respond appropriately with the subordinate meaning of the words.

The second main limitation relates to the clinical evaluation of the schizophrenic patients, which focuses on their thought disorders. We decided to proceed in this way in light of the relation between thought disorders and the abnormalities of semantic priming in schizophrenia. However, it also appears necessary to explore the psychotic symptomatology of such patients because the severity of their symptoms (negative and/or positive) may be a confounding variable.

Third, the effect of treatment (neuroleptics, both typical and atypical, and anticholinergics, etc) should be evaluated.

Last, the role of disorganization symptoms was tested in connection with the TLC scores, but no significant correlations were found. This is probably because of the low scores obtained by the schizophrenic patients on the TLC (Table 1) and is at odds with studies that have shown that only thoughtdisordered schizophrenic patients present difficulties in the processing of contextual semantic information. ${ }^{3,13}$

Our results, although obtained in small samples of patients, are encouraging if we wish to develop new methods for the remediation of contextual semantic processing in schizophrenia that are more ecological and better adapted to daily life. At an even simpler level, our results could help guide clinicians during interviews with schizophrenic patients to ensure better contextualization of what is said. Future studies with a more appropriate clinical design and involving a neuropsychological evaluation (executive 
functions, working memory) are necessary to determine the efficacy of the remediation of context processing.

\section{Acknowledgment}

Funding to support this study was awarded to CB-R by the Institut Universitaire de France, Ministère de l'Enseignement Supérieur et de la Recherche, Paris, France. Our thanks go to Dr Boisrenoult for his encouragement to write this article. We are grateful to Tim Pownall for reviewing the English.

\section{Disclosure}

The authors report no conflicts of interest in this work.

\section{References}

1. Baez S, Herrera E, Villarin L, et al. Contextual social cognition impairments in schizophrenia and bipolar disorder. PLOS ONE. 2013;8(3): e57664.

2. McClure MM, Barch DM, Flory JD, Harvey PD, Siever LJ. Context processing in schizotypal personality disorder: evidence of specificity of impairment to the schizophrenia spectrum. J Abnorm Psychol. 2008;117(2):342-354.

3. Bazin N, Perruchet P, Hardy-Bayle MC, Feline A. Context-dependent information processing in patients with schizophrenia. Schizophr Res. 2000;45(1-2):93-101.

4. Chung YS, Mathews JR, Barch DM. The effect of context processing on different aspects of social cognition in schizophrenia. Schizophr Bull. 2011;37(5):1048-1056.

5. Richard AE, Carter CS, Cohen JD, Cho RY. Persistence, diagnostic specificity and genetic liability for context-processing deficits in schizophrenia. Schizophr Res. 2013;147(1):75-80.

6. Bazin N, Perruchet P. Implicit and explicit associative memory in patients with schizophrenia. Schizophr Res. 1996;22(3):241-248.

7. Cohen JD, Servan-Schreiber D. Context, cortex, and dopamine: a connectionist approach to behavior and biology in schizophrenia. Psychol Rev. 1992;99(1):45-77.

8. Hardy-Baylé MC, Sarfati Y, Passerieux C. The cognitive basis of disorganization symptomatology in schizophrenia and its clinical correlates: toward a pathogenetic approach to disorganization. Schizophr Bull. 2003;29(3):459-471.

9. Kuperberg GR, Kreher DA, Ditman T. What can Event-related Potentials tell us about language, and perhaps even thought, in schizophrenia? Int J Psychophysiol. 2010;75(2):66-76.

10. Champagne-Lavau M, Charest A, Anselmo K, Rodriguez JP, Blouin G. Theory of mind and context processing in schizophrenia: the role of cognitive flexibility. Psychiatry Res. 2012;200(2-3):184-192.

11. Meyer DE, Schvaneveldt RW. Facilitation in recognizing pairs of words: evidence of a dependence between retrieval operations. J Exp Psychol. 1971;90(2):227-234.

12. Pomarol-Clotet E, Oh TM, Laws KR, McKenna PJ. Semantic priming in schizophrenia: systematic review and meta-analysis. Br J Psychiatry. 2008;192(2):92-97.
13. Besche-Richard C, Passerieux C. Semantic context-processing deficit in thought-disordered schizophrenic patients: evidence from new semantic priming paradigms. Cogn Neuropsychiatry. 2003;8(3):173-189.

14. Titone D, Levy DL, Holzman PS. Contextual insensitivity in schizophrenic language processing: evidence from lexical ambiguity. J Abnorm Psychol. 2000;109(4):761-767.

15. Chapman LJ, Chapman JP, Daut RL. Schizophrenic inability to disattend from strong aspects of meaning. J Abnorm Psychol. 1976;85(1): $35-40$.

16. Ketteler D, Theodoridou A, Ketteler S, Jäger M. High order linguistic features such as ambiguity processing as relevant diagnostic markers for schizophrenia. Schizophr Res Treatment. 2012;2012:825050.

17. Salisbury D. N400 to lexical ambiguity and semantic incongruity in schizophrenia. Int J Psychophysiol. 2010;75(2):127-132.

18. Salisbury DF, O'Donnell BF, McCarley RW, Nestor PG, Shenton ME. Event-related potentials elicited during a context-free homograph task in normal versus schizophrenic subjects. Psychophysiology. 2000; 37(4):456-463

19. Blairy S, Neumann A, Nutthals F, Pierret L, Collet D, Philippot P. Improvements in autobiographical memory in schizophrenia patients after a cognitive intervention: a preliminary study. Psychopathology. 2008; 41(6):388-396

20. Marsh PJ, Luckett G, Russell T, Coltheart M, Green MJ. Effects of facial emotion recognition remediation on visual scanning of novel face stimuli. Schizophr Res. 2012;141(2-3):234-240.

21. Statucka M, Walder DJ. Efficacy of social cognition remediation programs targeting facial affect recognition deficits in schizophrenia: a review and consideration of high-risk samples and sex differences. Psychiatry Res. 2013;206(2-3):125-139.

22. American Psychiatric Association. Diagnostic and Statistical Manual of Mental Disorders, 4th edition, text revised. 2000. Washington, DC: APA.

23. Deltour JJ. Échelle de vocabulaire Mill Hill. Paris: Les Éditions du Centre de Psychologie Appliquée; 2005.

24. Beck AT, Steer RA, Ball R, Ranieri W. Comparison of Beck Depression Inventories-IA and -II in psychiatric outpatients. J Pers Assess. 1996; 67(3):588-597.

25. Andreasen NC, Grove WM. Thought, language, and communication in schizophrenia: diagnosis and prognosis. Schizophr Bull. 1986;12(3): 348-359.

26. Bazin N, Lefrere F, Passerieux C, Sarfati Y, Hardy-Baylé MC. Troubles formels de la pensée: traduction française de l'échelle d'évaluation de la pensée, du langage et de la communication [Formal thought disorders: French translation of the Thought, Language and Communication assessment scale]. Encephale. 2002;28(2):109-119. French.

27. Camion J. Dictionnaire des homonymes de la langue française. Leuven, Belgium: Peeters Publishers; 1986.

28. Besche-Richard C, Passerieux C, Hardy-Baylé MC. Double-decision lexical tasks in thought-disordered schizophrenic patients: a path towards cognitive remediation? Brain Lang. 2005;95(3):395-401.

29. Kayser N, Sarfati Y, Besche C, Hardy-Baylé MC. Elaboration of a rehabilitation method based on a pathogenetic hypothesis of "theory of mind" impairment in schizophrenia. Neuropsychol Rehabil. 2006; 16(1):83-95.
Neuropsychiatric Disease and Treatment

\section{Publish your work in this journal}

Neuropsychiatric Disease and Treatment is an international, peerreviewed journal of clinical therapeutics and pharmacology focusing on concise rapid reporting of clinical or pre-clinical studies on a range of neuropsychiatric and neurological disorders. This journal is indexed on PubMed Central, the 'PsycINFO' database and CAS,

\section{Dovepress}

and is the official journal of The International Neuropsychiatric Association (INA). The manuscript management system is completely online and includes a very quick and fair peer-review system, which is all easy to use. Visit http://www.dovepress.com/testimonials.php to read real quotes from published authors. 